\title{
Multiplicity distribution of gluons in pQCD
}

\author{
G. H. Arakelyan ${ }^{1, a}$, Yu. M. Shabelski ${ }^{2, b}$, A. G. Shuvaev ${ }^{2, c}$ \\ ${ }^{1}$ A.Alikhanyan National Scientific Laboratory (Yerevan Physics Institute), 0036 Yerevan, Armenia \\ ${ }^{2}$ Kurchatov National Research Center, Petersburg Nuclear Physics Institute, Gatchina, St. Petersburg 188300, Russia
}

Received: 23 March 2020 / Accepted: 16 June 2020 / Published online: 29 June 2020

(C) The Author(s) 2020

\begin{abstract}
The multiplicity distribution of the gluons produced at the high energy is evaluated in BFKL approach. The distribution has Poisson form that can explain experimentally observed KNO scaling.

1. The multiple secondary particles production at high energy hadron-hadron collisions is known to exhibit KNO scaling [1] confirmed in many experimental papers [2,3]. The scaling means that the ratio $P_{n}(s)=\sigma_{n}(s) / \sigma_{i n}(s)$ of the cross section to produce $n$ secondaries in the collision event to the total inelastic cross section depends on the energy $\sqrt{s}$ only through the averaged multiplicity $\bar{n}, P_{n}(s)=1 / \bar{n} \Psi(n / \bar{n})$.

According to Reggeon calculus the high energy hadron scattering is mediated by the Pomerons exchange, the Pomeron being described by the cylinder type diagrams $[4,5]$. The inelastic amplitudes come about from the $s$ channel cutting of one or several Pomerons [6]. Each cut can be associated with the creation of two quark-gluon strings [8] whose subsequent hadronization gives rise to the multiple hadron production.
\end{abstract}

The observed KNO scaling has been explained $[8,10]$ by the various number of cut Pomerons (quark-gluon strings) that may contribute to the interaction amplitude. Assuming Poisson distribution for the secondaries produced from a single Pomeron the net sum over the cuts yields the function $\Psi(n / \bar{n})$. In general it is not of the Poisson type, its form is much more complicated. It is nevertheless $s$ independent in the rather wide energy interval although the scaling should be violated at the very high energies in agreement with the experimental data $[10,12]$.

There has been much theoretical activity in studying KNO scaling in multiplicity distribution of QCD parton cascades. The cascade starts off a primary virtual gluon whose virtuality $Q^{2}$ is of the order of the typical hard scale for the process, say, $e^{+} e^{-}$annihilation. The primary gluon emits secondary

\footnotetext{
a e-mail: argev@mail.yerphi.am

b e-mail: shabelsk@thd.pnpi.spb.ru

c e-mail: shuvaev@thd.pnpi.spb.ru (corresponding author)
}

gluon jets each of which, in turn, emits another jets and so on. The cascade development is described by the evolution equation that collects the leading or subleading powers of $\log Q^{2}$ (see e.g. [13], or [14] for review).

The task we are going to deal with here is alternative in that we treat the secondaries arising from the large $s$ scattering of almost on shell particles rather than from the decay of an virtual object. That is why the context of Regge theory, collecting the powers of $\log s$ or, equivalently, $\log 1 / x$ for the deep inelastic scattering, seems to be quite natural. An important point to be questioned at first is what kind of distribution could be expected from a single Pomeron, or in another language, from a single quark-gluon string. Is it evident that it is Poisson like? Some insight can be gained by calculating the distribution of perturbative gluons originating from BFKL Pomeron. It is the issue that is addressed below. 2.The BFKL Pomeron arises as a compound state of two reggeized gluons in the sum of ladder type diagrams [15, $16,18,20]$. The treatment essentially relies on Multi-Regge form of many-particle amplitudes. It provides an expression for the amplitude to produce $n$ gluons or, more generally $n$ jets, in the inelastic scattering, $A+B \rightarrow A^{\prime}+B^{\prime}+n$, at the large invariant energy $s=\left(p_{A}+p_{B}\right)^{2}$ and the kinematics, when the rapidity intervals between the jets are much larger those between the jets' constituents - multi-Regge kinematics (see [22] and references therein). This amplitude is actually nothing else as the cut through the Reggeon. Using $s$-channel unitarity it gives the imaginary part of the elastic scattering amplitude, which value at $t=0$ is translated into the total cross section,

$$
\sigma_{A B}(s)=\frac{1}{4} \frac{1}{(2 \pi)^{2}} \int \frac{d^{2} q}{q^{2}} \int \frac{d^{2} l}{l^{2}} \Phi_{A}(q)\langle q|G(Y)| l\rangle \Phi_{B}(l) .
$$

Here $\Phi_{A, B}$ are the color-singlet impact-factors, $Y=\ln s / s_{0}$ $\left(s_{0}\right.$ is an appropriate energy scale usually assumed in the Regge theory to be of the order of $\left.1 \mathrm{GeV}^{2}\right), G(Y)$ is the Green 
function of two interacting reggeized gluons. It is given by the series $[23,25]$

$$
\begin{aligned}
G(Y)= & e^{\Omega Y}+\sum_{n=1}^{\infty} \int_{0}^{Y} d y_{1} e^{\Omega\left(Y-y_{1}\right)} K_{r} \\
& \times \int_{0}^{y_{1}} d y_{2} e^{\Omega\left(y_{1}-y_{2}\right)} K_{r} \cdots \int_{0}^{y_{n-1}} d y_{n} e^{\Omega\left(y_{n-1}-y_{n}\right)} K_{r} e^{\Omega y_{n}},
\end{aligned}
$$

with the operators $\Omega$ and $K_{r}$ acting in the transverse momentum space. The operator $K_{r}$ describes the emission of real (on-shell) gluons,

$\left\langle q\left|K_{r}\right| l\right\rangle=\frac{g^{2} N_{c}}{(2 \pi)^{3}} \frac{2}{(q-l)^{2}+m_{g}^{2}}$,

the virtual corrections are collected in the gluons' trajectories, $\Omega=\omega_{1}+\omega_{2}$. They are equal for the total transferred momentum $q=0, \omega_{1}=\omega_{2}=\omega$,

$\langle q|\omega| l\rangle=-\frac{1}{2} \frac{g^{2} N_{c}}{(2 \pi)^{3}} \int d^{2} k \frac{q^{2}}{k^{2}(q-k)^{2}+m_{g}^{2}} \delta^{(2)}(q-l)$,

the gluon mass $m_{g}^{2}$ being the infrared cutoff.

The formulae (3), (4) are relevant in the Leading Logarithm Approximation (LLA) collecting the leading powers of $\alpha_{S} \ln s / s_{0}$, where $\alpha_{S}=g^{2} / 4 \pi, g$ is QCD coupling constant. The dominant contribution to cross sections of hard processes comes in LLA from multi-Regge kinematics, when the emitted particles are single gluons. The situation allowing for the emission of the jets made up of two or more particles corresponds to the next to the leading order corrections [23,26].

There is an equivalent form of the operators (3), (4), valid for the small $m_{g}$ [25],

$$
\begin{aligned}
K_{r} & =\alpha\left[-\ln m_{g}^{2} x^{2}+2 \ln 2+2 \psi(1)\right], \quad \omega \\
& =-\frac{1}{2} \alpha \ln \frac{q^{2}}{m_{g}^{2}},
\end{aligned}
$$

$\alpha \equiv N_{c} \frac{g^{2}}{4 \pi^{2}}, \psi(z)=\frac{d}{d z} \ln \Gamma(z)$. The variables $x_{k}$ are the transverse space coordinates conjugated to the momenta $q_{k}$ (the indices $k=1,2$ label the vectors' component in the transverse space), $q^{2}=q_{1}^{2}+q_{2}^{2}, x^{2}=x_{1}^{2}+x_{2}^{2}$. These variables are treated in the formula (5) as operators $\hat{q}_{k}, \hat{x}_{k}=i \frac{\partial}{\partial q_{k}}$ acting in the transverse space, $|l\rangle$ being the operator $\hat{q}_{k}$ eigenstates, $\hat{q}_{k}|l\rangle=l_{k}|l\rangle,\langle q \mid l\rangle=\delta^{(2)}(q-l)$. The operator $\omega=\omega\left(\hat{q}_{1}, \hat{q}_{2}\right)$ is diagonal in this basis, its matrix elements are given in (4), the expression (3) presents the matrix elements of the non diagonal operator $K_{r}=K_{r}\left(\hat{x}_{1}, \hat{x}_{2}\right)$. Hereafter we omits hats above the operators' symbols.

The series (2) is summed up as

$G(Y)=e^{K Y}, \quad K=K_{r}+\omega_{1}+\omega_{2}$, which is the operator form of the BFKL equation [25]. The mass $m_{g}$ cancels out in the operator $K$ for the color singlet Pomeron channel (as is evident from (5)).

The $N$-th order term in the expansion (2) describes the emission of $N$ real gluons. To pick up its relative weight in $G(Y)$ we multiply it by an auxiliary variable $u^{N}$, or in other words replace $K_{r} \rightarrow u K_{r}$. Then the probability to emit $n$ real gluons reads

$P_{N}=\left.\frac{1}{P(1)} \frac{1}{N !} \frac{\partial^{N}}{\partial u^{N}}\right|_{u=0} P(u)$,

with the generating function $P(u)$ obtained by modifying the formula (1),

$P(u)=\int \frac{d^{2} q}{q^{2}} \int \frac{d^{2} l}{l^{2}} \Phi_{A}(q)\left\langle q\left|e^{Y\left(u K_{r}+2 \omega\right)}\right| l\right\rangle \Phi_{B}(l)$.

To work out this expression we firstly rearrange the operator appearing in $P(u)$ as

$$
\begin{aligned}
K(u)= & u K_{r}+2 \omega=\alpha\left[u\left(-\ln x^{2}-\ln q^{2}\right)\right. \\
& \left.-\bar{u} \ln q^{2}+\bar{u} \ln m_{g}^{2}+2 u(\ln 2+\psi(1))\right],
\end{aligned}
$$

$\bar{u} \equiv 1-u$, and pass to the complex variables,

$$
\begin{aligned}
q & =q_{1}+i q_{2}, \quad q^{*}=q_{1}-i q_{2}, \quad q^{2}=q q^{*}, \quad x^{2} \\
& =-4 \frac{\partial}{\partial q} \frac{\partial}{\partial q^{*}},
\end{aligned}
$$

the hermitian conjugate being $\left[\frac{\partial}{\partial q}\right]^{+}=-\frac{\partial}{\partial q^{*}}$. Using the identity $[24,25]$

$$
\ln q+\ln \frac{\partial}{\partial q}=\frac{1}{2}\left[\psi\left(1+q \frac{\partial}{\partial q}\right)+\psi\left(-q \frac{\partial}{\partial q}\right)\right]
$$

and its hermitian conjugate we arrive at the form that falls into the holomorphic and antiholomorphic pieces,

$$
\begin{aligned}
K(u)= & \alpha \frac{1}{2} u\left[\psi(1+D)+\psi(-D)+\psi\left(1+D^{*}\right)+\psi\left(-D^{*}\right)\right] \\
& +\alpha L(u), \\
D \equiv & q \frac{\partial}{\partial q}, \quad D^{*} \equiv q^{*} \frac{\partial}{\partial q^{*}}, \\
& +2 u \psi(1) .
\end{aligned}
$$

Given the commutator $[\ln q, D]=-1$, it is easy to check another operator identity,

$e^{\frac{u}{\bar{u}} Q}[-\bar{u} \ln q] e^{-\frac{u}{\bar{u}} Q}=-\bar{u} \ln q-\frac{1}{2} u[\psi(1+D)+\psi(-D)]$

with the operator

$Q=\frac{1}{2} \ln \frac{\Gamma(1+D)}{\Gamma(-D)}, \quad Q^{*}=\frac{1}{2} \ln \frac{\Gamma\left(1+D^{*}\right)}{\Gamma\left(-D^{*}\right)}$.

Putting it together with the same identity for the antiholomorphic $\ln q^{*}$ results into the relation

$e^{\frac{u}{\bar{u}}\left(Q+Q^{*}\right)} \alpha L(u) e^{-\frac{u}{\bar{u}}\left(Q+Q^{*}\right)}=K(u)$, 
that in turn amounts to

$e^{Y K(u)}=e^{\frac{u}{\bar{u}}\left(Q+Q^{*}\right)} e^{\alpha Y L(u)} e^{-\frac{u}{\bar{u}}\left(Q+Q^{*}\right)}$.

Now one has to plug the identity (10) into the matrix element occurring in the function $P(u)(8)$. We do it in the following way

$$
\begin{aligned}
& \left\langle q\left|e^{\frac{u}{\bar{u}}\left(Q+Q^{*}\right)} e^{\alpha Y L(u)} e^{-\frac{u}{\bar{u}}\left(Q+Q^{*}\right)}\right| l\right\rangle \\
& =\langle q| \underbrace{e^{\frac{1}{2} \alpha Y L(u)} e^{-\frac{1}{2} \alpha Y L(u)}}_{1} e^{\frac{u}{u}\left(Q+Q^{*}\right)} e^{\frac{1}{2} \alpha Y L(u)} e^{\frac{1}{2} \alpha Y L(u)} e^{-\frac{u}{\bar{u}}\left(Q+Q^{*}\right)} \\
& \quad \times \underbrace{e^{-\frac{1}{2} \alpha Y L(u)} e^{\frac{1}{2} \alpha Y L(u)}|l\rangle}_{1}
\end{aligned}
$$

By elaborating on the identities

$$
e^{ \pm \frac{1}{2} \alpha Y L(u)} D e^{\mp \frac{1}{2} \alpha Y L(u)}=D \pm \frac{1}{2} \alpha Y \bar{u}
$$

along with the same for $D^{*}$ and

$$
\begin{aligned}
& \langle q| e^{\frac{1}{2} \alpha Y L(u)}=\langle q| e^{-\frac{1}{2} \alpha Y \bar{u} \ln \frac{q^{2}}{m_{g}^{2}}+\alpha Y u \psi(1)}, \\
& e^{\frac{1}{2} \alpha Y L(u)}|l\rangle=e^{-\frac{1}{2} \alpha Y \bar{u} \ln \frac{l^{2}}{m_{g}^{2}}+\alpha Y u \psi(1)}|l\rangle
\end{aligned}
$$

the function $P(u)$ is brought to the form

$$
\begin{aligned}
P(u)= & e^{2 \alpha Y u \psi(1)} \int \frac{d^{2} q}{q^{2}} \int \frac{d^{2} l}{l^{2}} \\
& \times \Phi_{A}(q)\left(\frac{q^{2}}{m_{g}^{2}}\right)^{-\frac{1}{2} \alpha Y \bar{u}} \Phi_{B}(l)\left(\frac{l^{2}}{m_{g}^{2}}\right)^{-\frac{1}{2} \alpha Y \bar{u}} \\
& \times\left\langle q\left|H\left(u, D, D^{*}\right)\right| l\right\rangle \\
H\left(u, D, D^{*}\right)= & \exp \left\{\frac { 1 } { 2 } \frac { \overline { u } } { \operatorname { u } } \left[\ln \frac{\Gamma\left(1-\frac{1}{2} L \bar{u}+D\right)}{\Gamma\left(\frac{1}{2} L \bar{u}-D\right)}\right.\right. \\
& +\ln \frac{\Gamma\left(1-\frac{1}{2} L \bar{u}+D^{*}\right)}{\Gamma\left(\frac{1}{2} L \bar{u}-D^{*}\right)} \\
& -\ln \frac{\Gamma\left(1+\frac{1}{2} L \bar{u}+D\right)}{\Gamma\left(-\frac{1}{2} L \bar{u}-D\right)} \\
& \left.\left.-\ln \frac{\Gamma\left(1+\frac{1}{2} L \bar{u}+D^{*}\right)}{\Gamma\left(-\frac{1}{2} L \bar{u}-D^{*}\right)}\right]\right\}
\end{aligned}
$$

This form has a merit of the infrared cutoff explicitly factorized out. Besides, the operator in the matrix element is diagonal in the basis, where $D$ and $D^{*}$ are diagonal,

$$
\langle q \mid v, n\rangle=\frac{1}{2 \pi}\left(q^{2}\right)^{-\frac{1}{2}+i \frac{1}{2} \nu+i n \phi},
$$

$-\infty<v<\infty, n$ is integer, $0<\phi<2 \pi$ is the polar angle in the transverse $q$-space,

$$
\begin{aligned}
&\left\langle v, n\left|H\left(u, D, D^{*}\right)\right| v^{\prime}, n^{\prime}\right\rangle=H(u, v, n) \delta\left(v-v^{\prime}\right) \delta_{n, n^{\prime}} \\
& H(u, v, n)= H\left(u, D \rightarrow \frac{1}{2}(-1+i v+n), D^{*}\right. \\
&\left.\rightarrow \frac{1}{2}(-1+i v-n)\right) .
\end{aligned}
$$

From now on we suppose the impact factors to be angular independent in the transverse space. Then after inserting the complete set,

$\sum_{n} \int_{-\infty}^{\infty} d v\left\langle q_{2} \mid v, n\right\rangle\left\langle v, n \mid q_{1}\right\rangle=\delta^{(2)}\left(q_{2}-q_{1}\right)$,

into the matrix element (11) only the terms with $n=0$ survive in $P(u)$. Suppose also $q_{R}$ is a typical momentum scale for the both impact factors, $\Phi_{A, B}=\Phi_{A, B}\left(q^{2} / q_{R}^{2}\right)$ and introduce the functions $C_{A, B}(n)$ according to the relation

$$
\int \frac{d^{2} q}{q^{2}} \Phi_{A, B}\left(\frac{q^{2}}{q_{R}^{2}}\right)\left(q^{2}\right)^{n}=\left(q_{R}^{2}\right)^{n} C_{A, B}(n) .
$$

With these notations one gets

$$
\begin{aligned}
P(u)= & e^{2 L u \psi(1)}\left(\frac{q_{R}^{2}}{m_{g}^{2}}\right)^{-L \bar{u}} \int d v C_{A}\left(-\frac{1}{2} L \bar{u}+\frac{1}{2} i v\right) \\
& \times C_{B}\left(-\frac{1}{2} L \bar{u}-\frac{1}{2} i v\right) H(u, v, 0)
\end{aligned}
$$

The function $H(u, v, 0)$ embodies the scattering dynamics. Taking the limit $\bar{u} \rightarrow 0$ we see that,

$$
P(1)=e^{2 L \psi(1)} \int \frac{d^{2} q}{q^{2}} \int \frac{d^{2} l}{l^{2}} \Phi_{A}(q)\left\langle q\left|e^{K(1) L}\right| l\right\rangle \Phi_{B}(l)
$$

with

$K(1)=-\frac{1}{2}\left[\psi(1+D)+\psi(-D)+\psi\left(1+D^{*}\right)+\psi\left(-D^{*}\right)\right]$

and consequently $e^{2 L \psi(1)} H(1, v, 0)=e^{\omega(v) L}$, where BFKL eigenvalue,

$\omega(v)=\alpha\left[2 \psi(1)-\psi\left(\frac{1}{2}+\frac{1}{2} i v\right)-\psi\left(\frac{1}{2}-\frac{1}{2} i v\right)\right]$,

yields for $v=0$ the Pomeron mediated elastic cross section. The main contribution for $L \gg 1$ comes to the integral from the region near $v=0, P(1) \sim C_{A}(0) C_{B}(0) \exp \left(\omega_{P} Y\right)$, where $\omega_{P}=\omega(0)=4 \alpha \ln 2$ is the LLA Pomeron intercept.

The function $P(u)(15)$ turns out, however, to be strongly suppressed when $m_{g} \rightarrow 0$ at least for $u<1$. On the other 
hand, the mean gluon number

$$
\bar{N}=\left.\frac{1}{P(1)} \frac{\partial}{\partial u} P(u)\right|_{u=1} \sim \ln \frac{q_{R}^{2}}{m_{g}^{2}},
$$

indicates the infinite growth of the emitted gluons with decreasing infrared cut off $m_{g}$. The reason behind this is in the virtual gluons. The function $P(u)$ is constructed in (15) to fix the number of the real gluons whereas the virtual ones associated with the gluon trajectory $\omega$ remain unrestricted. As a consequence we are dealing with the amplitudes of the given perturbation order for the real emission allowing at the same time for the virtual corrections of an arbitrary order. Different number of the real and virtual gluons, the latter number being unbounded while the first one is finite, breaks down the infrared cancelation. A possible way to improve it is to modify the operator $K(u) \rightarrow K(u, v)=u K_{r}+2 v \omega$ by adding a new variable $v$ that "counts" the virtual gluons. It simply results into redefinition $\bar{u} \rightarrow v-u$ in the all above expressions, so that $u$ and $\bar{u}$ become independent. Putting $m_{g} \rightarrow 0$ enforces $u=v$, that leaves us with $\bar{u} \rightarrow 0$ limit,

$P(u)=C_{A}(0) C_{B}(0) e^{\omega_{P} Y u}$.

written here for the dominant, $v=0$, part. Thus we arrive at the Poisson distribution,

$P_{N}=\frac{1}{N !}\left(\omega_{P} Y\right)^{N} e^{-\omega_{P} Y}$

This formula however is hardly directly applied to the observable emission. The infrared cancelation of real and virtual parts occurs in each perturbation order that is why we actually get here the distribution over the evolution steps rather than the emitted gluons. Put somewhat differently, it is the distribution of the ladder cells number in the Pomeron diagram for the exclusive cross section, which is finite for $m_{g} \rightarrow 0$. Dealing with inclusive processes one has to appreciate that there is always a physical infrared cut-off provided either by a sort of minimal experimentally resolved momentum or some kinematical restrictions imposed to select the proper observables. Thus we have to modify our treatment to accomodate the additional constraints.

3. First, we modify the expression (11) to get the distribution of the gluons produced with a given total transverse momentum $Q$. On this purpose the probability to emit $N$ gluons with transverse momenta $k_{i}$ has to be multiplied by the factor

$\delta^{(2)}\left(Q-\sum_{i=1}^{N} k_{i}\right)=\int \frac{d^{2} z}{(2 \pi)^{2}} e^{i z Q} \prod_{i=1}^{N} e^{-i z k_{i}}$

that results into modification of the real emission operator (3),

$\left\langle q\left|K_{r}\right| q^{\prime}\right\rangle \rightarrow e^{-i z\left(q-q^{\prime}\right)}\left\langle q\left|K_{r}\right| q^{\prime}\right\rangle$.
It leads to the replacement $\ln m_{g}^{2} x^{2} \rightarrow \ln m_{g}^{2}(x-z)^{2}$ in the formula (5). Then the equalities

$$
\begin{aligned}
\ln (x-z)^{2}= & 2 \ln 2+\ln \left(\frac{\partial}{\partial q}+\frac{i}{2} z^{*}\right) \\
& +\left[\ln \left(\frac{\partial}{\partial q}+\frac{i}{2} z^{*}\right)\right]^{+}, \quad z=z_{1}+i z_{2},
\end{aligned}
$$

and the chain of relations

$$
\begin{aligned}
\ln q & +\ln \left(\frac{\partial}{\partial q}+\frac{i}{2} z^{*}\right) \\
= & e^{-\frac{1}{2} i z^{*} q}\left[\ln q+\ln \frac{\partial}{\partial q}\right] e^{\frac{1}{2} i z^{*} q} \\
= & e^{-\frac{1}{2} i z^{*} q}\left[\frac{1}{2} \psi\left(1+q \frac{\partial}{\partial q}\right)\right. \\
& \left.+\frac{1}{2} \psi\left(-q \frac{\partial}{\partial q}\right)\right] e^{\frac{1}{2} i z^{*} q}=\frac{1}{2}\left[\psi\left(1+D_{z}\right)+\psi\left(-D_{z}\right)\right], \\
D_{z} & =q\left(\frac{\partial}{\partial q}+\frac{i}{2} z\right), \quad\left[\ln q, D_{z}\right]=-1
\end{aligned}
$$

together with its Hermitian conjugate and the equality

$$
\begin{aligned}
\left\langle q\left|H\left(u, D_{z}, D_{z}^{*}\right)\right| l\right\rangle & =\left\langle q\left|e^{-i q z} H\left(u, D, D^{*}\right) e^{i q z}\right| l\right\rangle \\
& =e^{i z(-q+l)}\left\langle q\left|H\left(u, D, D^{*}\right)\right| l\right\rangle
\end{aligned}
$$

yield (11) with the replacement

$$
\left\langle q\left|H\left(u, D, D^{*}\right)\right| l\right\rangle \rightarrow \int \frac{d^{2} z}{(2 \pi)^{2}} e^{i z(Q-q+l)}\left\langle q\left|H\left(u, D, D^{*}\right)\right| l\right\rangle .
$$

The second modification is the gap assumed in the outgoing gluons momentum spectrum. We pick up the processes, where all the gluons are produced with the transverse momentum $q_{i}$ larger than a certain value $q_{0}$. To fulfil the latter requirement one has to modify the real emission part in (3), $\left\langle q\left|K_{r}\right| q^{\prime}\right\rangle \rightarrow \theta\left(\left(q-q^{\prime}\right)^{2}-q_{0}^{2}\right)\left\langle q\left|K_{r}\right| q^{\prime}\right\rangle$, or, within logarithmic accuracy, $\ln m_{g}^{2} x^{2} \rightarrow \ln q_{0}^{2} x^{2}$ in the formula (5). The logarithmic accuracy implies the value $q_{0}$ to be small compared to the typical momentum scale, $q_{0}^{2} \ll q_{R}^{2}$. The power-like corrections beyond this region make the relevant operators to be non diagonal in the $|v, n\rangle$ basis, which invalidates the method performance.

The third modification is that the secondaries are registered not at all kinematically available rapidities $0<y<Y$ but in a more narrow range $0<y_{1}<y<y_{2}<Y$. To implement this condition into generating function one has to replace $K \rightarrow K(u)(9)$ at the interval $\left[y_{1}, y_{2}\right]$ to fix there the number of emitted particles, whereas the intervals $\left[0, y_{1}\right]$ and $\left[y_{2}, Y\right]$. have to be left with unchanged BFKL operator $K=K(1)$ (16). Thus instead of (8) one has

$$
\begin{aligned}
P(u)= & \int \frac{d^{2} q}{q^{2}} \int \frac{d^{2} l}{l^{2}} \\
& \times \Phi_{A}(q)\left\langle q\left|e^{K(1)\left(Y-y_{2}\right)} e^{K(u)\left(y_{2}-y_{1}\right)} e^{K(1) y_{1}}\right| l\right\rangle \Phi_{B}(l) .
\end{aligned}
$$


To proceed further, we first substitute the operator in the middle with the above obtained expressions (11) and (12),

$$
\begin{aligned}
& \left\langle q\left|e^{K(1)\left(Y-y_{2}\right)} e^{K(u)\left(y_{2}-y_{1}\right)} e^{K(1) y_{1}}\right| l\right\rangle \\
& =e^{L u\left(2 \psi(1)-\ln \frac{q_{0}^{2}}{m_{g}^{2}}\right)}\langle q| e^{K(1)\left(Y-y_{2}\right)}\left(\frac{q^{2}}{m_{g}^{2}}\right)^{-\frac{1}{2} L \bar{u}} H\left(u, D, D^{*}\right) \\
& \quad \times\left(\frac{q^{2}}{m_{g}^{2}}\right)^{-\frac{1}{2} L \bar{u}} e^{K(1) y_{1}}|l\rangle .
\end{aligned}
$$

Here $L=\alpha\left(y_{2}-y_{1}\right)$. The next step is purely algebraic. With the help of identities

$$
D q^{n}=q^{n}(D+n), \quad D^{*}\left(q^{*}\right)^{n}=\left(q^{*}\right)^{n}\left(D^{*}+n\right),
$$

valid for any $n$, we drag BFKL exponents with $K(1)$ operator to the right and to the left through $q^{2}$ powers until they join the operator $H\left(u, D, D^{*}\right)$,

$$
\begin{aligned}
& e^{K(1)\left(Y-y_{2}\right)}\left(\frac{q^{2}}{m_{g}^{2}}\right)^{-\frac{1}{2} L \bar{u}} H\left(u, D, D^{*}\right)\left(\frac{q^{2}}{m_{g}^{2}}\right)^{-\frac{1}{2} L \bar{u}} e^{K(1) y_{1}} \\
& =\left(\frac{q^{2}}{m_{g}^{2}}\right)^{-\frac{1}{2} L \bar{u}} e^{K_{L}\left(Y-y_{2}\right)} H\left(u, D, D^{*}\right) e^{K_{R} y_{1}}\left(\frac{q^{2}}{m_{g}^{2}}\right)^{-\frac{1}{2} L \bar{u}}, \\
& K_{L}=-\frac{1}{2}\left[\psi\left(1+D+\frac{1}{2} L \bar{u}\right)+\psi\left(-D-\frac{1}{2} L \bar{u}\right)\right. \\
& \left.\quad+\psi\left(1+D^{*}+\frac{1}{2} L \bar{u}\right)+\psi\left(-D^{*}-\frac{1}{2} L \bar{u}\right)\right] \\
& K_{R}=-\frac{1}{2}\left[\psi\left(1+D-\frac{1}{2} L \bar{u}\right)\right. \\
& \quad+\psi\left(-D+\frac{1}{2} L \bar{u}\right)+\psi\left(1+D^{*}-\frac{1}{2} L \bar{u}\right) \\
& \left.\quad+\psi\left(-D^{*}+\frac{1}{2} L \bar{u}\right)\right] .
\end{aligned}
$$

The third step is to insert the complete set,

$$
\begin{aligned}
P(u)= & \sum_{n} \int d v \int \frac{d^{2} q}{q^{2}} \Phi_{A}(q)\left\langle q\left|\left(\frac{q^{2}}{m_{g}^{2}}\right)^{-\frac{1}{2} L \bar{u}}\right| v, n\right\rangle \\
& \times\left\langle v, n\left|e^{K_{L}\left(Y-y_{2}\right)} H\left(u, D, D^{*}\right) e^{K_{R} y_{1}}\right| v, n\right\rangle \\
& \times \int \frac{d^{2} l}{l^{2}}\left\langle v, n\left|\left(\frac{q^{2}}{m_{g}^{2}}\right)^{-\frac{1}{2} L \bar{u}}\right| l\right\rangle \Phi_{B}(l) .
\end{aligned}
$$

Here we take into account that the operators $K_{L}, K_{R}, H$ are diagonal in $|\nu, n\rangle$ basis, besides the angular independence of the impact factors selects $n=0$ in the sum. Recalling (13),
(14) the momentum integrals read

$$
\begin{aligned}
& \int \frac{d^{2} q}{q^{2}} \Phi_{A, B}(q)\left\langle q\left|\left(\frac{q^{2}}{m_{g}^{2}}\right)^{-\frac{1}{2} L \bar{u}}\right| v, 0\right\rangle \\
& =C_{A, B}\left(-\frac{1}{2} L \bar{u} \pm \frac{1}{2} i v\right)\left(q_{R}^{2}\right)^{-\frac{1}{2} \pm \frac{1}{2} i v}\left(\frac{q_{R}^{2}}{m_{g}^{2}}\right)^{-\frac{1}{2} L \bar{u}}
\end{aligned}
$$

Thus we have

$$
P(u)=\int d v e^{-L \ln \frac{q_{R}^{2}}{m_{g}^{2}}} e^{L \omega_{0} u} T(u), \quad \omega_{0}=\ln \frac{q_{R}^{2}}{q_{0}^{2}}+2 \psi(1),
$$

where

$$
\begin{aligned}
T(u)= & \int d v C_{A}\left(-\frac{1}{2} L \bar{u}+\frac{1}{2} i v\right) C_{B}\left(-\frac{1}{2} L \bar{u}-\frac{1}{2} i v\right) \\
& \times\left\langle v, 0\left|e^{K_{L}\left(Y-y_{2}\right)} H\left(u, D, D^{*}\right) e^{K_{R} y_{1}}\right| v, 0\right\rangle .
\end{aligned}
$$

Notice that $u$-independent multiplies are irrelevant in the generating function as they are absorbed in its overall normalization. That is why the fictitious gluon mass $m_{g}$ drops out of the final distribution. The natural infrared cut off is provided by $q_{0}$ momentum. In what follows we will assume the typical scale $q_{R}^{2} \gg q_{0}^{2}$

If the value $\omega_{0}$ can be treated as a large parameter the function $P(u)$ results into Poisson distribution. It clearly follows from (22) by keeping there the maximal $\omega_{0}$ power for each $N$, which amounts to differentiating $e^{L \omega_{0} u}$ only while the rest factors are taken just at $u=0$. The mean gluon number then $\bar{N}=\omega_{0} L \gg 1$. The first correction arises from the terms with one power of $\omega_{0}$ less than in the main order,

$$
\begin{aligned}
P_{N} & =P_{N}^{(0)}+P_{N}^{(1)} \\
& =\left.C \frac{1}{N !} \frac{\partial^{N}}{\partial u^{N}} P(u)\right|_{u=0} \\
& \approx C \frac{1}{N !}\left(\omega_{0} L\right)^{N} T(0)\left[1+N \frac{1}{\omega_{0} L} \frac{T^{\prime}(0)}{T(0)}\right] \\
& \approx C \frac{1}{N !}\left(\omega_{0} L\right)^{N} T(0)\left[1+\frac{1}{\omega_{0} L} \frac{T^{\prime}(0)}{T(0)}\right]^{N}
\end{aligned}
$$

where $C$ is the normalization constant. As is evident from the last line the first order correction preserves the form of the Poisson distribution,

$P_{N}=\frac{1}{N !}\left(\omega_{0}^{\prime} L\right)^{N} e^{-\omega_{0}^{\prime} L}$,

affecting only the mean number of gluons, $\bar{N}^{\prime}=\omega^{\prime} L=$ $\omega_{0} L+\frac{T^{\prime}(0)}{T(0)}$.

We try to estimate the first order contribution for $L \gg 1$, supposing, in addition, both the rapidity intervals to be equal (actually of the same order) and large, $\left(Y-y_{2}\right)=y_{1}=y$, $\alpha y \gg 1$. 
The leading for $L \gg 1$ terms could be expected to arise from the function $H(u, v, 0)$, therefore the part of interest is

$$
\begin{aligned}
T^{\prime}(0) \approx & \int d v C_{A}\left(-\frac{1}{2} L+\frac{1}{2} i v\right) C_{B}\left(-\frac{1}{2} L-\frac{1}{2} i v\right) \\
& \times\left.\exp \left\{\alpha y\left(K_{L}(0, v)+K_{R}(0, v)\right)\right\} \frac{\partial}{\partial u} H(u, v, 0)\right|_{u=0}
\end{aligned}
$$

It is convenient to rewrite the sum in the exponent as

$$
\begin{aligned}
K_{L}(0, v)+K_{R}(0, v)= & -\psi\left(\frac{1}{2}+\frac{1}{2} i v+\frac{1}{2} L\right) \\
& -\psi\left(\frac{1}{2}-\frac{1}{2} i v+\frac{1}{2} L\right) \\
& -\frac{\pi \sin \pi L}{\cos \pi L+\cosh 2 \pi \nu} .
\end{aligned}
$$

Aiming at the large $L$ asymptotics, it is sufficient to take this expression only for integer $L$, that removes at all the last term, whereupon we have

$$
\begin{aligned}
K_{L}(0, v)+K_{R}(0, v)= & -2 \psi\left(\frac{1}{2}+\frac{1}{2} L\right) \\
& +\frac{1}{4} \psi^{\prime \prime}\left(\frac{1}{2}+\frac{1}{2} L\right) v^{2}+O\left(v^{4}\right) .
\end{aligned}
$$

Similarly presenting the function

$$
\begin{aligned}
& \left.\frac{\partial}{\partial u} H(u, v, 0)\right|_{u=0} \\
& =-2 \ln \Gamma\left(\frac{1}{2}+\frac{1}{2} L-\frac{1}{2} i v\right)-2 \ln \Gamma\left(\frac{1}{2}+\frac{1}{2} L+\frac{1}{2} i v\right) \\
& \quad-\ln (\cos \pi L+\cosh 2 \pi v)+\ln 2 \pi^{2}
\end{aligned}
$$

makes it clear that only the first two terms grow with $L$ while the last ones, being periodic in $L$, do not and can be omitted. Given now that $\psi^{\prime \prime}(x) \sim-1 / x^{2}$ for $x \gg 1$ the typical $v$ values in the $T(0)$ integral (23), $v^{2} \sim L^{2} /(\alpha y)$, are small compared to $L^{2}$ when $\alpha y \gg 1$. It allows to neglect $v$ in the function $H^{\prime}(0, v, 0)$ as well as in the impact factor functions $C_{A, B}$ in (24), that finally yields

$\frac{T^{\prime}(0)}{T(0)}=-L \ln \frac{L}{2}+L+O(1)$

It is worth to point out that in terms of the original basic decomposition (11) the main contribution to the multiplicity is due to the power multipliers whereas the function $H\left(u, D, D^{*}\right)$ specifies the corrections.
The generating function for the distribution of the gluons carrying in aggregate the fixed total transverse momentum $Q$ according to (11) and (19) is

$$
\begin{aligned}
P(Q, u)= & \int \frac{d^{2} z}{(2 \pi)^{2}} e^{L u\left(2 \psi(1)-\ln \frac{q_{0}^{2}}{m_{g}^{2}}\right)} e^{i z Q} P(z, u), \\
P(z, u)= & \int \frac{d^{2} q}{q^{2}} \int \frac{d^{2} l}{l^{2}} \\
& \times \Phi_{A}(q)\left(\frac{q^{2}}{m_{g}^{2}}\right)^{-\frac{1}{2} L \bar{u}}\left\langle q\left|e^{-i q z} H\left(u, D, D^{*}\right) e^{i q z}\right| l\right\rangle \\
& \times\left(\frac{l^{2}}{m_{g}^{2}}\right)^{-\frac{1}{2} L \bar{u}} \Phi_{B}(l) .
\end{aligned}
$$

The momentum $Q$ is the total momentum of the gluons that are supposed to be registered, that is only those emitted with the transverse momenta larger than $q_{0}$.

There is an important difference from the previous case, where the main order source is in the terms with no derivatives of the function $H\left(u, D, D^{*}\right)$. Here the dominant terms including $H\left(0, D, D^{*}\right)=1$ result into $z$-independent contribution that in turn produces $P(Q, u) \sim \delta^{(2)}(Q)$.

To find the leading behavior at small $q_{0}$ and the total momentum $Q \neq 0$ we apply the formal trick:

$H\left(u, D, D^{*}\right)=\left.H\left(u, \frac{\partial}{\partial t}, \frac{\partial}{\partial t^{*}}\right) e^{t D} e^{t^{*} D^{*}}\right|_{t=t^{*}=0}$,

and employ the operator identities

$$
\begin{gathered}
e^{t D} e^{t^{*} D^{*}} e^{i q z}=e^{i \bar{q} z} e^{t D} e^{t^{*} D^{*}}, \bar{q}_{1}=\frac{1}{2}\left(t+t^{*}\right) q_{1}-\frac{1}{2 i}\left(t-t^{*}\right) q_{2}, \\
\bar{q}_{2}=\frac{1}{2 i}\left(t-t^{*}\right) q_{1}+\frac{1}{2}\left(t+t^{*}\right) q_{2}, \\
e^{t D} e^{t^{*} D^{*}} \int d^{2} l|l\rangle \frac{1}{l^{2}} \Phi_{B}\left(\frac{l^{2}}{q_{R}^{2}}\right)=\int d^{2} l|l\rangle \frac{e^{-t-t^{*}}}{l^{2}} \Phi_{B}\left(e^{t+t^{*}} \frac{l^{2}}{q_{R}^{2}}\right) .
\end{gathered}
$$

Then we get

$$
\begin{aligned}
P(z, u)= & \left.H\left(u, \frac{\partial}{\partial t}, \frac{\partial}{\partial t^{*}}\right)\right|_{t=t^{*}=0} e^{-\left(t+t^{*}\right)\left(\frac{1}{2} L \bar{u}+1\right)} \\
& \times \int \frac{d^{2} q}{q^{4}} e^{-i(q-\bar{q}) z}\left(\frac{q^{2}}{m_{g}^{2}}\right)^{-L \bar{u}} \\
& \times \Phi_{A}\left(\frac{q^{2}}{q_{R}^{2}}\right) \Phi_{B}\left(e^{t+t^{*}} \frac{q^{2}}{q_{R}^{2}}\right)
\end{aligned}
$$

or doing $z$ integrals, that return $(2 \pi)^{2} \delta^{(2)}(Q-q+\bar{q})$,

$$
\begin{aligned}
& P(Q, u)=e^{-L \ln \frac{Q^{2}}{m_{g}^{2}}} e^{L\left(2 \psi(1)+\ln \frac{Q^{2}}{q_{0}^{2}}\right) u} \\
& \left.H\left(u, \frac{\partial}{\partial t}, \frac{\partial}{\partial t^{*}}\right)\right|_{t=t^{*}=0} e^{-\left(t+t^{*}\right)\left(\frac{1}{2} L \bar{u}+1\right)} n_{t, t^{*}}^{L \bar{u}-1} \\
& \quad \times \frac{1}{Q^{4}} \Phi_{A}\left(\frac{1}{n_{t, t^{*}}} \frac{Q^{2}}{q_{R}^{2}}\right) \Phi_{B}\left(\frac{e^{t+t^{*}}}{n_{t, t^{*}}} \frac{Q^{2}}{q_{R}^{2}}\right) \\
& n_{t, t^{*}} \equiv\left(1-e^{t}\right)\left(1-e^{t^{*}}\right) .
\end{aligned}
$$


In principle this form could offer one more tool to handle the generating function, but here it is only needed to factorize out the leading asymptotics assuming $\omega_{0}(Q)=2 \psi(1)+$ $\ln \frac{Q^{2}}{q_{0}^{2}} \gg 1$. The non vanishing result is obtained after the function $H$ is once differentiated,

$P_{N}^{(1)}(Q)=e^{-L\left(\ln \frac{q_{R}^{2}}{m_{g}^{2}}+\ln \frac{Q^{2}}{q_{R}^{2}}\right)} C_{1}\left(\frac{Q^{2}}{q_{R}^{2}}\right) \frac{1}{(N-1) !} \omega_{0}^{N-1}(Q)$.

Although this is a first term it looks like a correction to the Poisson distribution, the computation of $C_{1}$ function being similar to what has been done above for $T^{\prime}(0)$. The analog of the leading term comes from the delta-function piece,

$$
\begin{aligned}
P_{N}^{(0)}(Q) & =e^{-L\left(\ln \frac{q_{R}^{2}}{m_{g}^{2}}\right)} C_{0} \frac{1}{N !}\left(\omega_{0} L\right)^{N} \delta^{(2)}(Q), \\
C_{0} & =\frac{1}{q_{R}^{2}} \int d^{2} x \Phi_{A}(x) \Phi_{B}(x)\left(x^{2}\right)^{-L-2} .
\end{aligned}
$$

If the total momentum essential range is $q_{0}^{2} \ll Q^{2} \ll q_{R}^{2}$ (due to impact factors) the value $C_{1}\left(\frac{Q^{2}}{q_{R}^{2}}\right) \approx C_{1}(0)$ could be found from the relation

$$
\begin{gathered}
\int d^{2} Q\left[P_{N}^{(0)}(Q)+P_{N}^{(1)}(Q)\right] \\
=e^{-L \ln \frac{q_{R}^{2}}{m_{g}^{2}}\left[\frac{1}{N !}\left(\omega_{0} L\right)^{N} T(0)\right.} \\
\left.+\frac{1}{(N-1) !}\left(\omega_{0} L\right)^{N-1} T^{\prime}(0)\right]
\end{gathered}
$$

for known $T(0), T^{\prime}(0)$.

4. Concluding, the distribution of the gluons arising from the cut of BFKL Pomeron in LLA has been found for the two separate cases. The first one is entirely unobservable although it literally refers to the gluons "inside" the Pomeron, that is to the distribution of the ladder diagrams in the Pomeron Green function. It is exactly of the Poisson type, the "mean number" of the gluons, or the ladder "length", being proportional to the Pomeron intercept.

The second case concerns the real multiple emission in the scattering. The gluon distribution is generally more complex but getting closer to the Poisson one, when the gap parameter $q_{0}$, that plays the role of the infrared cut off, decreases. However in this case the mean gluon number turns out not to be identical to the intercept.

The first order corrections to the Poisson distribution obtained for $L=\alpha Y \gg 1$ affects only its mean number parameter. The distribution of the gluons with a fixed total transverse momentum looks like the first order correction with the main term vanishing for the non zero momentum value.

The next order corrections as well as the effects from $L \sim 1$ presumably cause the deviation from the Poisson distribution but its form would be more dependent on the impact factors. Here we are mainly interested in the effects of BFKL dynamics and have used roughly estimated impact factors.

This situation looks different compared to $e^{+} e^{-}$case, where more complicated parton distributions are obtained [13]. Assuming soft branching hypotheses [27] telling that main qualitative features of the secondary hadrons distribution are similar to those of the partons one plausibly expects the secondary hadron to be distributed similarly to the gluons according to Poisson form. It could explain the observed KNO scaling effects $[1,5]$.

The authors are grateful to M.G. Ryskin for helpful discussion.

Data Availability Statement This manuscript has no associated data or the data will not be deposited. [Authors' comment: The main result here is Poisson distribution confirmed for the secondary gluons at high $s$ scattering and specified with a single parameter, mean gluon number, there are no more special data to be associated with.]

Open Access This article is licensed under a Creative Commons Attribution 4.0 International License, which permits use, sharing, adaptation, distribution and reproduction in any medium or format, as long as you give appropriate credit to the original author(s) and the source, provide a link to the Creative Commons licence, and indicate if changes were made. The images or other third party material in this article are included in the article's Creative Commons licence, unless indicated otherwise in a credit line to the material. If material is not included in the article's Creative Commons licence and your intended use is not permitted by statutory regulation or exceeds the permitted use, you will need to obtain permission directly from the copyright holder. To view a copy of this licence, visit http://creativecomm ons.org/licenses/by/4.0/.

Funded by $\mathrm{SCOAP}^{3}$.

\section{References}

1. Z. Koba, H.B. Nielsen, P. Olesen, Nucl. Phys. B 40, 372 (1972)

2. G.J. Alner et al. (UA5 Collaboration), Phys. Lett. B 138, 304 (1984)

3. R.E. Ansorge et al. (UA5 Collaboration), Z. Phys. C 43, 357 (1989)

4. M. Ciafaloni, G. Marchesini, G. Veneziano, Nucl. Phys. B 98, 472 (1975)

5. A.B. Kaidalov, K.A. Ter-Martirosian, Phys. Lett. 117B, 247 (1982)

6. V.A. Abramovsky, V.N. Gribov, O.V. Kancheli, Yad. Fiz. 18, 595 (1973)

7. V.A. Abramovsky, V.N. Gribov, O.V. Kancheli, Sov. J. Nucl. Phys. 18, 308 (1974)

8. A.B. Kaidalov, K.A. Ter-Martirosian, Sov. J. Nucl. Phys. 39, 979 (1984)

9. A.B. Kaidalov, K.A. Ter-Martirosian, Yad. Fiz. 39, 1545 (1984)

10. A.B. Kaidalov, K.A. Ter-Martirosyan, Sov. J. Nucl. Phys. 40, 135 (1984)

11. A.B. Kaidalov, K.A. Ter-Martirosyan, Yad. Fiz. 40, 211 (1984)

12. S. Hegyi, Nucl. Phys. Proc. Suppl. 92, 122 (2001). arXiv:hep-ph/0011301

13. Y.L. Dokshitzer, Phys. Lett. B 305, 295 (1993)

14. Y.L. Dokshitzer, V.A. Khoze, A.H. Mueller, S.I. Troian, Basics of perturbative QCD (Frontieres, Gif-sur-Yvette, 1991) 
15. V.S. Fadin, E.A. Kuraev, L.N. Lipatov, Multi-Regge form of manyparticle amplitudes. Phys. Lett. B 60, 50 (1975)

16. E.A. Kuraev, L.N. Lipatov, V.S. Fadin, Zh Eksp, Teor. Fiz. 71, 840 (1976)

17. E.A. Kuraev, L.N. Lipatov, V.S. Fadin, Sov. Phys. JETP 44, 443 (1976)

18. E.A. Kuraev, L.N. Lipatov, V.S. Fadin, Zh Eksp, Teor. Fiz. 72, 377 (1977)

19. E.A. Kuraev, L.N. Lipatov, V.S. Fadin, Sov. Phys. JETP 45, 199 (1977)

20. I.I. Balitsky, L.N. Lipatov, Sov. J. Nucl. Phys. 28, 822 (1978)

21. I.I. Balitsky, L.N. Lipatov, Yad. Fiz. 28, 1597 (1978)

22. V.S. Fadin, R. Fiore, Phys. Lett. B 440, 359 (1998)

23. V.S. Fadin, R. Fiore, M.G. Kozlov, A.V. Reznichenko, Phys. Lett. B 639, 74 (2006). arXiv:hep-ph/0602006
24. H.J. de Vega, L.N. Lipatov, Phys. Lett. B 578, 335 (2004). arXiv:hep-ph/0310124

25. B.L. Ioffe, V.S. Fadin, L.N. Lipatov, Quantum Chromodynamics Perturbative and Nonperturbative Aspects (Cambridge University Press, Cambridge, 2010). (ISBN 9781107424753)

26. V.S. Fadin, M.G. Kozlov, A.V. Reznichenko, Phys. Rev. D 92(8), 085044 (2015). arXiv: 1507.00823 [hep-th]

27. Y.L. Dokshitzer, V.S. Fadin, V.A. Khoze, Z. Phys. C 18, 37 (1983) 